https://doi.org/10.15407/ujpe64.3.217

V.I. ROMANENKO, ${ }^{1}$ N.V. KORNILOVSKA ${ }^{2}$

${ }^{1}$ Institute of Physics, Nat. Acad. of Sci. of Ukraine

(46, Nauky Ave., Kyiv 03680,Ukraine; e-mail:vr@iop.kiev.ua)

${ }^{2}$ Kherson National Technical University

(24, Berislavske Highway, Kherson 73008, Ukraine)

\title{
ON THE ACCURACY OF ERROR PROPAGATION CALCULATIONS BY ANALYTIC FORMULAS OBTAINED FOR THE INVERSE TRANSFORMATION
}

\begin{abstract}
The accuracy of error propagation calculations is estimated for the transformation $x \rightarrow y=$ $f(x)$ of the normally distributed random variable $x$. The estimation is based on the formulas for the error propagation obtained for the inverse transformation $y \rightarrow x$ of the normally distributed random variable $y$. In the general case, the calculation accuracy for the mean value and the variance of the random variable $y$ is shown to be of the first order of magnitude in the variance of the random variable $x$.
\end{abstract}

Ke ywords: error propagation, variance, mean value, normal distribution.

\section{Introduction}

When analyzing the results of a physical experiment, it is often necessary to calculate their accuracy by proceeding from the known accuracy of measured quantities. For example, in the X-ray diffraction analysis, the measured diffraction angles of X-rays are used to determine the crystal lattice constants. The formulas used for the calculation of the accuracy of determined values are known well. They provide an excellent result, if the error (mean-square deviation $\sigma_{x}$ ) of the measured quantity $x$ is so small that the dependence of the calculated result on $x$, i.e. $y=f(x)$, can be considered to be a linear function within the limits of this error [1]. However, it is quite possible that the dispersion of the measured quantity is rather large. As a result, the estimate of the error for the $y$ value calculated on the basis of experimental data can significantly change.

If the quantity $x$ is characterized by a distribution function $F(x)$, for which the integrals $\int_{-\infty}^{\infty} x^{n} F(x) d x$ and $\int_{-\infty}^{\infty} f(x)^{n} F(x) d x(n=1,2)$ can be calculated analytically, the mean value (mathematical expectation) and the variance of the quantity $y$ can be expressed in terms of the parameters of the distribution $F(x)$. In this case, we can express, in principle, the mathematical expectation $\bar{y}$ and the variance $\sigma_{y}^{2}$ of

(c) V.I. ROMANENKO, N.V. KORNILOVSKA, 2019

ISSN 2071-0194. Ukr. J. Phys. 2019. Vol. 64, No. 3 the quantity $y$ via the mathematical expectation $\bar{x}$ and the variance $\sigma_{x}^{2}$ of the quantity $x$. Such calculations were made, e.g., for the dependences $y=\cos x$ [2] and $y=x^{2}[3]$ in the case of normal distribution for $x$, and for the dependence $y=\sqrt{x}[4]$ in the case of standard (with the zero mathematical expectation) normal distribution for $x$. However, if the indicated integrals cannot be calculated analytically, but the integrals $\int_{-\infty}^{\infty} y^{n} G(y) d y$ and $\int_{-\infty}^{\infty} g(y)^{n} G(y) d y(n=$ $1,2)$ can be, where $g(y)=x$ is a function inverse to $y=f(x)$, and $G(y)$ is the distribution function for $y$, we again obtain relations that connect the pair $\bar{x}$ and $\sigma_{x}^{2}$ with the pair $\bar{y}$ and $\sigma_{y}^{2}[2,3]$. This procedure was done in work [2] for $y=\arccos (x)$ and work [3] for $y=\sqrt{x}$ (inverse transformations look like $x=\cos y$ and $x=y^{2}$, respectively).

It should be noted that if the function $f(x)$ - and, accordingly, $g(y)$ - is nonlinear, the expression for the distribution function of the random variable $y$ differs from that for the distribution of the random variable $x$. For instance, if the random variable $x$ is distributed according to the normal law, then the distribution of the random variable $y=f(x)$ is not normal. However, the author of works $[2,3]$ proposed the formulas obtained for the inverse transformation $y \rightarrow x$ under the assumption about the normal distribution of $y$ to be also used for the calculation of $\bar{y}$ and $\sigma_{y}^{2}$ at the transformation $x \rightarrow y=f(x)$ of the normally distributed quantity $x$, i.e. ignoring the fact that the distribution 
of $x$ is not normal for the $y \rightarrow x$ transformation. Unfortunately, the error arising due to the substitution of a non-normal $x$-distribution by the normal $y$-one was not discussed in the cited works. Nevertheless, since the general idea of the calculation method proposed in works $[2,3]$ seems interesting to us, it is worth estimating its error.

The paper structure is as follows. In Section 2, the stages of the general procedure used to calculate the accuracy of statistical parameters characterizing the distribution of the quantity $y=f(x)$ are listed. The calculation procedure itself is expounded in Section 3 in detail. In Section 4, an example of calculations for the transformation $f(x)=\sqrt{x}$ is given, and its comparison with the results of numerical calculations is made. A brief conclusion is formulated at the end of the paper.

\section{Calculation Scheme}

The accuracy of statistical characteristics $y=f(x)$ calculated by analytical formulas obtained for the inverse transformation $x=g(y)$ will be estimated using the following scheme.

1. A random variable $x$ is assumed to be characterized by the normal distribution with mean value $\bar{x}$ and variance $\sigma_{x}^{2}$. A quantity $y$ is related to $x$ by means of the relation $y=f(x)$. The latter can be expanded in a Taylor series.

2. The mean value $\bar{y}$ and variance $\sigma_{y}^{2}$ of the quantity $y$ are calculated.

3. Now, the random variable $y$ is assumed to be characterized by the normal distribution with mean value $\overline{y_{a}}$ and variance $\sigma_{y_{a}}^{2}$. The quantity $x$ is related to the quantity $y$ by the relation $x=g(y)$, which can also be expanded in a Taylor series.

4. The corresponding mean value $\overline{x_{a}}$ and variance $\sigma_{x_{a}}^{2}$ of the quantity $x$ are calculated. The obtained distribition of $x$ is obviously non-normal.

5. The values of $\overline{x_{a}}$ and $\sigma_{x_{a}}^{2}$, which characterize the non-normal distribution of the quantity $x$ for the transformation $y \rightarrow x=g(y)$ of the normally distributed quantity $y$, are identified with the values of $\bar{x}$ and $\sigma_{x}^{2}$. It is this identification that forms the basis of works [2,3] and gives rise to the accuracy restriction of the formulas quoted in those works.

6. Finally, the $\bar{y}$ - and $\sigma_{y}^{2}$-values are compared with the $\overline{y_{a}}$ - and $\sigma_{y_{a}}^{2}$-ones, respectively. The difference between them characterizes the error of the method proposed in works $[2,3]$ for the calculation of the mathe- matical expectation and the variance obtained at the transformation $x \rightarrow y=f(x)$ with the help of analytical formulas obtained for those quantities at the inverse transformation $y \rightarrow x=g(y)$ of the normally distributed quantity $y$.

\section{The Accuracy of Error \\ Propagation at the Transformation $x \rightarrow y$ on the Basis of the Results Obtained for the Transformation $y \rightarrow x$}

Let a random variable $x$ be characterized by a normal distribution function $F(x)$ with mean value $\bar{x}$ and variance $\sigma_{x}^{2}$ (i.e. $\sigma_{x}$ is the root-mean-square deviation):

$F(x)=\frac{1}{\sigma_{x} \sqrt{2 \pi}} \exp \left[-\frac{(x-\bar{x})^{2}}{2 \sigma_{x}^{2}}\right]$.

This distribution function satisfies the following formulas, in which $n$ is an integer:

$$
\begin{aligned}
& \int_{-\infty}^{\infty}(x-\bar{x})^{2 n+1} F(x) d x=0, \\
& \int_{-\infty}^{\infty}(x-\bar{x})^{2 n} F(x) d x=(2 n-1) ! ! \sigma_{x}^{2 n} \quad(n \geq 1), \\
& \int_{-\infty}^{\infty} F(x) d x=1 .
\end{aligned}
$$

The variables $y$ and $x$ are related to each other by the relation $y=f(x)$, which can be rewritten in the form of the Taylor series

$f(x)=\sum_{n=0}^{\infty} a_{n}(x-\bar{x})^{n}$,

where

$a_{n}=\left.\frac{1}{n !} \frac{d^{n}}{d x^{n}} f(x)\right|_{x=\bar{x}}$.

\subsection{Mean value and variance at the transformation $y=f(x)$}

Taking formulas (2) and (3) into account, we can determine the mean value $\bar{y}$ for the function $y=f(x)$, if the random variable $x$ is distributed according to law (1):

$\bar{y}=\int_{-\infty}^{\infty} f(x) F(x) d x=\sum_{n=0}^{\infty} a_{n} \int_{-\infty}^{\infty}(x-\bar{x})^{n} F(x) d x=$

ISSN 2071-0194. Ukr. J. Phys. 2019. Vol. 64, No. 3 
$=a_{0}+\sum_{n=1}^{\infty} a_{2 n}(2 n-1) ! ! \sigma_{x}^{2 n}$.

With the fourth-order accuracy in $\sigma_{x}$, we obtain

$\bar{y}=a_{0}+a_{2} \sigma_{x}^{2}+3 a_{4} \sigma_{x}^{4}$.

The variance of $y$ (mean square deviation from the mean value) equals

$\sigma_{y}^{2}=\int_{-\infty}^{\infty}(y-\bar{y})^{2} F(x) d x=\int_{-\infty}^{\infty} y^{2} F(x) d x-\bar{y}^{2}$.

The mean value of $y^{2}$,

$\overline{y^{2}}=\int_{-\infty}^{\infty} y^{2} F(x) d x$

can be written in the form

$\overline{y^{2}}=\sum_{n=0}^{\infty} \sum_{m=0}^{\infty} a_{n} a_{m} \int_{-\infty}^{\infty}(x-\bar{x})^{n+m} F(x) d x$

or, equivalenly,

$\overline{y^{2}}=\sum_{n=0}^{\infty} \sum_{m=n}^{\infty} a_{n} a_{m-n} \int_{-\infty}^{\infty}(x-\bar{x})^{m} F(x) d x$.

In view of formulas (2) and (3), equality (12) can also be expressed as

$\overline{y^{2}}=\sum_{n=0}^{\infty} \sum_{m=\left[\frac{n+1}{2}\right]}^{\infty} a_{n} a_{2 m-n} \int_{-\infty}^{\infty}(x-\bar{x})^{2 m} F(x) d x$.

Here, the square brackets mean the integer part of a number. The last expression can be written in the form

$\overline{y^{2}}=a_{0} a_{0}+\sum_{m=1}^{\infty} a_{0} a_{2 m} \int_{-\infty}^{\infty}(x-\bar{x})^{2 m} F(x) d x+$

$+\sum_{n=1}^{\infty} \sum_{m=\left[\frac{n+1}{2}\right]}^{\infty} a_{n} a_{2 m-n} \int_{-\infty}^{\infty}(x-\bar{x})^{2 m} F(x) d x$.

From whence, as well as from Eqs. (2) and (3), it follows that

$\overline{y^{2}}=a_{0} a_{0}+\sum_{m=1}^{\infty} a_{0} a_{2 m}(2 m-1) ! ! \sigma_{x}^{2 m}+$

ISSN 2071-0194. Ukr. J. Phys. 2019. Vol. 64, No. 3 $+\sum_{n=1}^{\infty} \sum_{m=\left[\frac{n+1}{2}\right]}^{\infty} a_{n} a_{2 m-n}(2 m-1) ! ! \sigma_{x}^{2 m}$.

Within an accuracy of $\sigma_{x}^{4}$, the latter expression looks like

$\overline{y^{2}}=a_{0} a_{0}+\left(2 a_{0} a_{2}+a_{1}^{2}\right) \sigma_{x}^{2}+3\left(2 a_{0} a_{4}+2 a_{1} a_{3}+a_{2}^{2}\right) \sigma_{x}^{4}$

With regard for Eqs. (8), (9), and (16), we obtain

$\sigma_{y}^{2}=a_{1}^{2} \sigma_{x}^{2}+2\left(a_{2}^{2}+3 a_{1} a_{3}\right) \sigma_{x}^{4}$.

The obtained expressions - formula (8) for $\bar{y}$ and formula (17) for $\sigma_{y}^{2}$ - are completely identical to those obtained for $f(x)=x^{2}$ in work [3] $\left(a_{0}=\bar{x}^{2}, a_{1}=2 \bar{x}\right.$, $\left.a_{2}=1, a_{3}=a_{4}=0\right)$ and identical with an accuracy of $\sigma_{x}^{4}$ to the results obtained for $f(x)=\cos x$ in work [2] $\left(a_{0}=\cos \bar{x}, a_{1}=-\sin \bar{x}, a_{2}=-\frac{1}{2} \cos \bar{x}\right.$, $\left.a_{3}=\frac{1}{6} \sin \bar{x}, a_{4}=\frac{1}{24} \cos \bar{x}\right)$.

\subsection{Mean value and variance at the transformation $x=g(y)$}

Now, let us consider the function $x=g(y)$, which is inverse to $y=f(x)$. The random variable $y$ is assumed to be distributed according to the normal law

$G(y)=\frac{1}{\sigma_{y_{a}} \sqrt{2 \pi}} \exp \left[-\frac{\left(y-\overline{y_{a}}\right)^{2}}{2 \sigma_{y_{a}}^{2}}\right]$

with the mean value $\overline{y_{a}}$ and the variance $\sigma_{y_{a}}^{2}\left(\sigma_{y_{a}}\right.$ is the root-mean-square deviation). Let the Taylor series expansion of the function $g(y)$ look like

$g(y)=\sum_{n=0}^{\infty} b_{n}\left(y-y_{0}\right)^{n}$

where

$b_{n}=\left.\frac{1}{n !} \frac{d^{n}}{d y^{n}} g(y)\right|_{y=y_{0}}, \quad y_{0}=f(\bar{x})=a_{0}$.

We should calculate the mean value $\overline{x_{a}}$ and the variance $\sigma_{x_{a}}^{2}$ of the values given by the function $x=g(y)$. Note that the point $y_{0}$, at which the function $g(y)$ was expanded in the Taylor series (19), does not coincide in the general case with the maximum of the distribution $G(y)$, which is achieved at $y=\overline{y_{a}}$. Therefore, it is convenient to write the function $g(y)$ in the form $g(y)=\sum_{n=0}^{\infty} b_{n}\left(y-\overline{y_{a}}+\delta\right)^{n}$, 
where $\delta=\overline{y_{a}}-y_{0}$, and to use the integrals

$$
\begin{aligned}
& \int_{-\infty}^{\infty}\left(y-\overline{y_{a}}\right)^{2 n+1} G(y) d y=0, \\
& \int_{-\infty}^{\infty}\left(y-\overline{y_{a}}\right)^{2 n} G(y) d y=(2 n-1) ! ! \sigma_{y_{a}}^{2 n}(n \geq 1), \\
& \int_{-\infty}^{\infty} G(y) d y=1,
\end{aligned}
$$

while calculating $\overline{x_{a}}$ and $\sigma_{x_{a}}^{2}$. To the fourth-order accuracy in $\sigma_{y_{a}}$, we obtain

$$
\begin{aligned}
& \overline{x_{a}}=\int_{-\infty}^{\infty} G(y) g(y) d y=b_{0}+b_{1} \delta+ \\
& +b_{2}\left(\delta^{2}+\sigma_{y_{a}}^{2}\right)+b_{3}\left(\delta^{3}+3 \delta \sigma_{y_{a}}^{2}\right)+ \\
& +b_{4}\left(\delta^{4}+6 \delta^{2} \sigma_{y_{a}}^{2}+3 \sigma_{y_{a}}^{4}\right) .
\end{aligned}
$$

The variance $\sigma_{x_{a}}^{2}$ is calculated by the formula

$\sigma_{x_{a}}^{2}=\overline{x_{a}^{2}}-{\overline{x_{a}}}^{2}$

where

$\overline{x_{a}^{2}}=\int_{-\infty}^{\infty} G(y) g(y)^{2} d y$.

Assuming $\delta$ to have the same order of smallness as $\sigma_{y_{a}}$, we obtain that, to the fourth-order accuracy in $\sigma_{y_{a}}$,

$\sigma_{x_{a}}^{2}=b_{1}^{2} \sigma_{y_{a}}^{2}+4 b_{1} b_{2} \delta \sigma_{y_{a}}^{2}+2 \sigma_{y_{a}}^{4}\left(3 b_{1} b_{3}+b_{2}^{2}\right)+$

$+2 \sigma_{y_{a}}^{2} \delta^{2}\left(3 b_{1} b_{3}+2 b_{2}^{2}\right)$.

\subsection{Relations for the coefficients in the Taylor series of the functions $y=f(x)$ and $x=g(y)$}

For further calculations, we have to find the relations between the collections of coefficients $\left\{b_{n}\right\}$ (n= $=1,2,3,4)$ and $\left\{a_{n}\right\}(n=1,2,3,4)$. The coefficients $a_{n}$ are determined by formula (6) via the derivatives of the function $f(x)$. The required relations can be found from the identity

$g(f(x))=x$.
The latter, being rewritten in terms of the Taylor series for the functions $f(x)$ and $g(x)$, reads

$\sum_{n=0}^{\infty} b_{n}\left(\sum_{m=0}^{\infty} a_{m}(x-\bar{x})^{m}-a_{0}\right)^{n}=x$.

By equating the terms with the same power exponents of $x$ in the left- and right-hand sides of Eq. (30), we obtain equations that determine the coefficients $\left\{b_{n}\right\}$ in terms of the known coefficients $\left\{a_{n}\right\}$. In calculations, it is convenient to introduce the variable $\xi=x-\bar{x}$ and to rewrite Eq. (30) in the form

$$
\sum_{n=0}^{\infty} b_{n}\left(\sum_{m=1}^{\infty} a_{m} \xi^{m}\right)^{n}=\bar{x}+\xi
$$

Now, we should equate the coefficients in the terms on the left- and right-hand sides of Eq. (31) with the same power exponents of $\xi$. The first five equalities look like

$b_{0}=\bar{x}$,

$b_{1} a_{1}=1$,

$a_{1}^{2} b_{2}+a_{2} b_{1}=0$,

$a_{1}^{3} b_{3}+2 a_{1} a_{2} b_{2}+a_{3} b_{1}=0$,

$a_{1}^{4} b_{4}+3 a_{1}^{2} a_{2} b_{3}+2 a_{1} a_{3} b_{2}+a_{2}^{2} b_{2}+a_{4} b_{1}=0$.

As a result, we obtain

$b_{0}=\bar{x}$,

$b_{1}=\frac{1}{a_{1}}$

$b_{2}=-\frac{a_{2}}{a_{1}^{3}}$,

$b_{3}=\frac{-a_{1} a_{3}+2 a_{2}^{2}}{a_{1}^{5}}$,

$b_{4}=\frac{-a_{1}^{2} a_{4}+5 a_{1} a_{2} a_{3}-5 a_{2}^{3}}{a_{1}^{7}}$.

\subsection{Calculation errors}

for the mean value and the variance obtained by the method of works [2,3]

In order to determine the calculation errors for $\bar{y}$ and $\sigma_{y}$, let us identify the quantities $\bar{x}_{a}$ and $\sigma_{x_{a}}$, which characterize the non-normal distribution of the variable $x$ after the transformation $y \rightarrow x=g(y)$ of the ISSN 2071-0194. Ukr. J. Phys. 2019. Vol. 64, No. 3 
normally distributed variable $y$, with $\bar{x}$ and $\sigma_{x}$, respectively. As was marked above, it is this equating that lies in the basis of works $[2,3]$ and gives rise to the accuracy restriction of the formulas presented there. Let us compare $\bar{y}$ with $\overline{y_{a}}$ and $\sigma_{y}^{2}$ with $\sigma_{y_{a}}^{2}$. The differences between those values will characterize the error of the method proposed in works $[2,3]$ to calculate the mathematical expectation and the variance of the random variable $y=f(x)$. Our calculations will be carried out with an accuracy of up to the fourthorder of smallness in $\sigma_{x}$.

The solutions $\delta$ and $\sigma_{y_{a}}$ of the equations

$$
\begin{aligned}
& \bar{x}=b_{0}+b_{1} \delta+b_{2}\left(\delta^{2}+\sigma_{y_{a}}^{2}\right)+b_{3}\left(\delta^{3}+3 \delta \sigma_{y_{a}}^{2}\right)+ \\
& +b_{4}\left(\delta^{4}+6 \delta^{2} \sigma_{y_{a}}^{2}+3 \sigma_{y_{a}}^{4}\right) \\
& \sigma_{x}^{2}=b_{1}^{2} \sigma_{y_{a}}^{2}+4 b_{1} b_{2} \delta \sigma_{y_{a}}^{2}+2 \sigma_{y_{a}}^{4}\left(3 b_{1} b_{3}+b_{2}^{2}\right)+ \\
& +2 \sigma_{y_{a}}^{2} \delta^{2}\left(3 b_{1} b_{3}+2 b_{2}^{2}\right)
\end{aligned}
$$

are sought in the form

$\delta=d_{1}+d_{2}+d_{3}+d_{4}$,

$\sigma_{y_{a}}^{2}=s_{2}+s_{4}$,

where the terms $d_{n}$ and $s_{n}$ are assumed to have the $\sigma_{x}^{n}$-order of smallness. Substituting Eqs. (44) and (45) into Eqs. (42) and (43), equating the coefficients in the terms with the same order of smallness on the left- and right-hand sides of the equations obtained, and taking Eqs. (37)-(41) into account, we arrive at

$d_{1}=0$,

$d_{2}=a_{2} \sigma_{x}^{2}$,

$d_{3}=0$,

$d_{4}=3 a_{4} \sigma_{x}^{4}-6 \frac{a_{2} a_{3}}{a_{1}} \sigma_{x}^{4}$,

$s_{2}=a_{1}^{2} \sigma_{x}^{2}$,

$s_{4}=6 a_{1} a_{3} \sigma_{x}^{4}-10 a_{2}^{2} \sigma_{x}^{4}$.

Accordingly, from Eqs. (42) and (43), we get

$\overline{y_{a}}=a_{0}+a_{2} \sigma_{x}^{2}+3 a_{4} \sigma_{x}^{4}-6 \frac{a_{2} a_{3}}{a_{1}} \sigma_{x}^{4}$,

$\sigma_{y_{a}}^{2}=a_{1}^{2} \sigma_{x}^{2}+6 a_{1} a_{3} \sigma_{x}^{4}-10 a_{2}^{2} \sigma_{x}^{4}$

By comparing Eqs. (52) and (53) with Eqs. (8) and (17), one can see that the errors calculated for the mean value and the variance of the random quantity $y$ connected with the normally distributed random variable $x$ by the relation $y=f(x)$ with the use of the method proposed in works $[2,3]$ are

$\overline{y_{a}}-\bar{y}=-6 \frac{a_{2} a_{3}}{a_{1}} \sigma_{x}^{4}$

$\sigma_{y_{a}}^{2}-\sigma_{y}^{2}=-12 a_{2}^{2} \sigma_{x}^{4}$.

\section{Discussion of the Results Obtained}

To illustrate the results obtained, let us compare the mean values and the variances of the random quantity $y=f(x)=\sqrt{x}$ calculated in different ways:

1. numerical integration in expressions (7) and (9);

2. using the formulas of work [3];

3. using the obtained formulas (8) and (17);

4. using the obtained formulas (52) and (53).

Furthermore, let us estimate the accuracy of the error propagation method, which was proposed in works [2,3], using formulas (54) and (55).

While calculating $\bar{y}, \overline{y_{a}}, \overline{\sigma_{y}^{2}}$, and $\overline{\sigma_{y_{a}}^{2}}$, we have to know the Taylor series expansion coefficients of the function $f(x)=\sqrt{x}$ in a vicinity of the mean value $\bar{x}$ of the random quantity $x$. Using Eq. (6), we obtain

$a_{0}=\sqrt{\bar{x}}$

$a_{1}=\frac{1}{2 \sqrt{\bar{x}}}$

$a_{2}=-\frac{1}{8 \bar{x} \sqrt{\bar{x}}}$

$a_{3}=\frac{1}{16 \bar{x}^{2} \sqrt{\bar{x}}}$

$a_{4}=-\frac{5}{128 \bar{x}^{3} \sqrt{\bar{x}}}$.

The formulas for the mean value and the variance obtained in work [3] are

$\overline{y_{\text {Rode }}}=\sqrt[4]{\bar{x}^{2}-\frac{1}{2} \sigma_{x}^{2}}$

$\sigma_{y_{\text {Rode }}}^{2}=\bar{x}-\sqrt{\bar{x}^{2}-\frac{1}{2} \sigma_{x}^{2}}$.

Let us compare these results with $\overline{y_{\text {Numerical }}}$ and $\overline{\sigma_{y_{\text {Numerical }}}^{2}}$ obtained by numerically integrating expressions (7) and (9) to an accuracy of $10^{-10}$, by putting $f(x)=\sqrt{|x|}$. Below, the results of a numerical integration will be referred to as "exact" ones. The error is determined as the difference between the calculated value and the corresponding result of a numerical integration.

In Table, the results of calculations of the mean value and the variance of the quantity $y$ after the transformation $y=\sqrt{x}$ of the random variable $x$ distributed according to Eq. (1) with $\bar{x}=25$ and $\sigma_{x}=5$ are quoted. As was expected, $\bar{y}$ and $\overline{\sigma_{y}^{2}}$ are the closest to the exact values. The calculation errors for $\overline{y_{\text {Rode }}}$ and $\overline{y_{a}}$ are approximately identical, as well as 
Calculation results for the mean value and variance variance of the quantity $y=\sqrt{x}$ at $\bar{x}=25$ and $\sigma_{x}=5$

\begin{tabular}{|c|c|c|}
\hline Quantity & Value & Error \\
\hline$\overline{y_{\text {Rode }}}$ & 4.9748103 & $8.7 \times 10^{-4}$ \\
\hline $\bar{y}$ & 4.9740625 & $1.2 \times 10^{-4}$ \\
\hline$\overline{y_{a}}$ & 4.9748125 & $8.7 \times 10^{-4}$ \\
\hline$\overline{\sigma_{y_{\text {Numerical }}}^{2}}$ & 0.2599280 & 0 \\
\hline$\overline{\sigma_{y_{\text {Rode }}}^{2}}$ & 0.2512627 & $-8.7 \times 10^{-3}$ \\
\hline$\overline{\sigma_{y}^{2}}$ & 0.2587500 & $-1.2 \times 10^{-3}$ \\
\hline$\overline{\sigma_{y_{a}}^{2}}$ & 0.2512500 & $-8.7 \times 10^{-3}$ \\
\hline
\end{tabular}

the calculation errors for $\overline{\sigma_{y_{\text {Rode }}}^{2}}$ and $\overline{\sigma_{y_{a}}^{2}}$. This means that expressions (52) for $\overline{y_{a}}$ and (53) for $\overline{\sigma_{y_{a}}^{2}}$ describe well dependences (61) and (62) obtained in work [3]. Their differences are $\overline{y_{\text {Rode }}}-\overline{y_{a}}=-2.2 \times 10^{-6}$ and $\overline{\sigma_{y_{\text {Rode }}}^{2}}-\overline{\sigma_{y_{a}}^{2}}=1.3 \times 10^{-5}$ for the indicated parameter values. The data presented in Table also demonstrate that the substitution of the normal distribution by a non-normal one, for which formulas (52), (53) and (61), (62) are valid - this procedure was made in works $[2,3]-$ inserts the errors $\overline{y_{a}}-\bar{y}=7.5 \times 10^{-4}$ and $\overline{\sigma_{y_{a}}^{2}}-\sigma_{y}^{2}=-7.5 \times 10^{-3}$.

Note that the illustrative example given in work [3] as an additional argument in favor of the error propagation method $[2,3]$ developed on the basis of inverse functions, for which integrals in Eqs. (7) and (9) can be calculated analytically, looks like a convincing one. The reason is very simple: the ratio between the parameters $\bar{x}=40.45$ and $\sigma_{x}=0.89$ used in calculations turns out rather small, $\sigma_{x} / \bar{x}=2.2 \times 10^{-2}$. In this case, one may expect that one or two terms in the series expansion of the function $f(x)=\sqrt{x}$ will suffice for a rather accurate calculation of the mean value and the variance. Our calculations for the same values of statistical parameters gave $\overline{y_{\text {Rode }}}-\overline{y_{\text {Numerical }}}=$ $=1.4 \times 10^{-7}$ and $\overline{\sigma_{y_{\text {Rode }}}^{2}}-\overline{\sigma_{y_{\text {Numerical }}}^{2}}=-1.8 \times 10^{-6}$.

\section{Conclusions}

In this work, the accuracy of the error propagation method proposed in works $[2,3]$, which was developed for the transformation $y=f(x)$ on the basis of formulas obtained for the inverse function $x=g(y)$ with the normal distribution of the random variable $y$, is analyzed. Our results testify that this method, generally speaking, has no advantages over the standard method - the expansion the function $f(x)$ into the Taylor series and the account for only the zeroth and first terms [1] - while calculating the variance of the random variable $y$. At the same time, it gives a more precise, with an accuracy of $\sigma_{x}^{2}$, mean value, which is equivalent to the account for the second term in the Taylor series. As one can see from formulas (52)-(55), the case where the second derivative of $f(x)$ at $x=\bar{x}$ vanishes is an exception, and the method proposed in works $[2,3]$ gives the average value and the variance with an accuracy of least $\sigma_{x}^{4}$.

1. D.J. Hudson. Lectures on Elementary Statistics and Probability (CERN, 1963).

2. G.G. Rode. Propagation of measurement errors and measured means of a physical quantity for the elementary functions $\cos x$ and $\arccos x$. Ukr. J. Phys. 61, 345 (2016).

3. G.G. Rode. Propagation of the measurement errors and measured means of physical quantities for the elementary functions $x^{2}$ and $\sqrt{x}$. Ukr. J. Phys. 62, 184 (2017).

4. P. Kosobutsky. Analytical relations for the mathematical expectation and variance of a standard distributed random variable subjected to the $\sqrt{x}$ transformation. Ukr. J. Phys. 63, 215 (2018).

Received 04.02.19.

Translated from Ukrainian by O.I. Voitenko

В.І. Романенко, Н.В. Корніловсъка

ПРО ТОЧНІСТЬ РОЗРАХУНКУ

ПЕРЕНЕСЕННЯ ПОХИБОК ЗА АНАЛІТИЧНИМИ ФОРМУЛАМИ ДЛЯ ОБЕРНЕНОГО ПЕРЕТВОРЕННЯ

Р ез ю м е

Оцінено точність розрахунку перенесення похибок при перетворенні $x \rightarrow y=f(x)$ при нормальному розподілі випадкової величини $x$ на основі формул для перенесення похибок, отриманих для оберненого перетворення $y \rightarrow x$ з розподіленою за нормальним законом випадковою величиною $y$. Показано, що у загальному випадку точність розрахунку середнього значення і дисперсії випадкової величини $y$ має перший порядок за дисперсією випадкової величини $x$. 